\title{
Article
}

\section{Improvement of Position Repeatability of a Linear Stage with Yaw Minimization}

\author{
Doo Hyun Cho ${ }^{1}$, Hyo Chan Kwon ${ }^{2}$ and Kwon Hee Kim ${ }^{3, *}$
}

1 Department of Mechanical Engineering, Graduate school, Korea University, 145, Anam-Ro, Seongbuk-gu, Seoul, 02841, Republic of Korea; dh9.cho@gmail.com

2 Department of Mechanical Design, Gyeonggi University of Science and Technology, Siheung, 15073, Republic of Korea; 20201006@gtec.ac.kr

3 Department of Mechanical Engineering, Korea University, 145, Anam-Ro, Seongbuk-gu, Seoul, 02841, Republic of Korea; kwonhkim@korea.ac.kr

* Correspondence: kwonhkim@korea.ac.kr

\begin{abstract}
Recently, due to the miniaturization of electronic products, printed circuit boards (PCBs) have also become smaller. This trend has led to the need for high-precision electrical test equipment to check PCBs for disconnections and short circuits. The purpose of this study is to improve the position repeatability of the platform unit up to $\pm 2.5 \mu \mathrm{m}$ in a linear stage type test equipment. For this purpose, the causes of position errors of the platform unit are analyzed. The platform unit holding the PCB is driven by a single-axis linear ball screw drive system offset from its geometric center due to design constraints. The yaw rotation of the platform is found to have a dominant effect on position repeatability. To address this problem, the methods of adding balancing weights to the platform unit and adjusting the stiffness of LM Guides are proposed. This reduces the yaw rotation by moving the centers of mass and stiffness closer to the linear ball screw actuator. In the verification tests, the position repeatability was decreased to less than $\pm 1.0 \mu \mathrm{m}$.
\end{abstract}

Keywords: Precision stage; Balanced platform; Balancing weight; Drive force offset; Yaw motion; Abbe error; Error prediction; Low-cost stage; Open frame stage; Linear motion guide (LM Guide); Python; GEKKO; ANSYS bushing joint

\section{Introduction}

Electrical test equipment measures the resistance of a circuit pattern on a printed circuit board (PCB) to inspect any abnormalities. Electrical inspection is performed with thousands or more probe pins concurrently positioned on the upper and lower surfaces of the PCB along the circuit check points. As the recent miniaturization and thinning of electronic products has resulted in fine-pitch patterned PCB, there is a need for the highprecision alignment of the probe pins. Lowering the manufacturing cost of the equipment is further required.

The electrical test equipment has an $x-y$ stage that moves the PCB to the position coordinates and precisely aligns the PCB with the probe pins. The stage is a module of significant importance that must meet productivity and precision. Figure 1 shows the schematic diagram of the $x-y$ stage.

The stage has a platform with central opening to allow the probe pins onto the upper/lower surfaces of the PCB. A coordinate system is chosen such that x-axis is in the length direction of the platform while the y-axis is in the width direction. The platform is driven by linear ball screw actuator systems offset from the platform center. Due to the offsets, yaw moments are generated during the operation of the stage. The major contributor to the yaw motion is driving force offset, resulting in deteriorated precision due to the Abbe error. [1] 


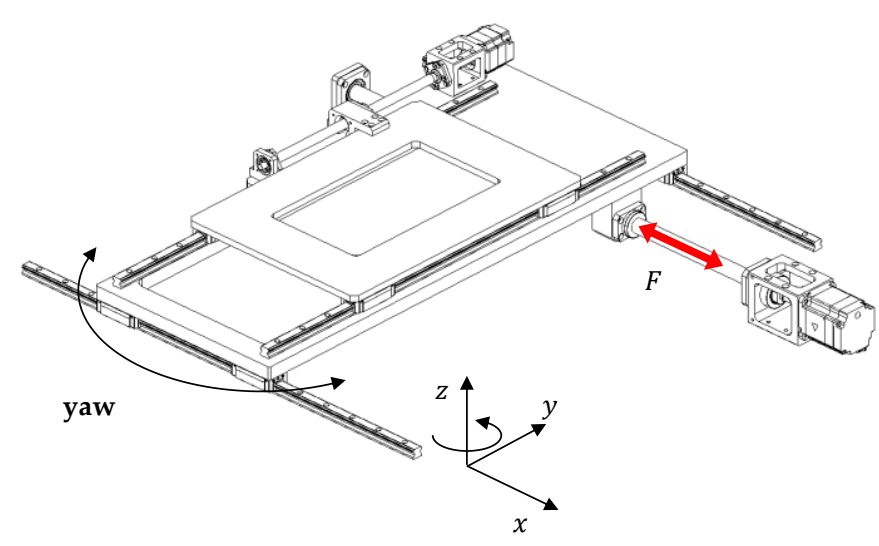

Figure 1. Schematic of offset driven x-y stage.

To reduce the yaw moment, the linear stage may be driven by synchronous control with driving units installed on both sides of the guide. Since the parallelism between the two linear motion guides (LM Guide) on both sides is not perfect, the actuator loads may vary during operation. Repeated load fluctuations may cause structural distortions over time which ultimately may lead to jamming. Thus, the synchronous control method has many shortcomings, such as increased manufacturing and maintenance costs and complexity of control programs compared to the single motor control method.

Typically, a single axis linear motion stage consists of a pair of LM Guides. Each rail of the configured LM Guides is exposed to shape errors, machining errors on the installation surface, and assembly errors. As a result, unintended 5-degree of freedom (DOF) error motions occur. Among these, the errors in the translational motion direction can be corrected to some extent by the averaging effect, whereas it is difficult to correct errors arising from the angular motions, such as yaw. [1,2]

Various studies have been conducted to analyze the error motions caused by the geometrical error of the LM guide's rail. Major studies have simplified the balls of the LM Guide, which are the most elastic parts deformed by force, to spring models. Such simplification has been used to predict the translational error motions of the stage with LM Guide rail shape error and ball stiffness. [3-16] Linear stages for electrical test machines are designed with high acceleration and deceleration capabilities for productivity. As a result, significant yaw motion and vibration of the platform could occur. Most of the previous studies, however, employed static equilibrium equations for the platform without inertial effects.

The precision level of the stage may be supplemented by measuring the motion error of the platform and compensating it with corrected position coordinates. For this purpose, the position repeatability of the platform is crucial. $[15,19]$. However, as in the x-axis shown in Figure 1, if yaw motion occurs, the position repeatability of the platform varies depending on the magnitude and direction of the driving force $F$ as well as the bearing friction between the platform and the rails. Since the friction is a result of complex tribological interaction between the balls and the rails subjected to statistical uncertainties [17, $21,22]$, platform yaw is most unpredictable. In this case, it is extremely difficult to enhance the position accuracy even with the compensations.

In this work, a rigid body dynamics model is proposed to analyze the contributions of various factors to platform yaw motion subjected to single offset driving force. Further, a method for minimizing the yaw motion is presented along with experimental verifications of its effectiveness. This effort led to the development of a low-cost precision stage satisfying the platform acceleration of $1 \mathrm{~g}$ and the position repeatability of $\pm 1.0 \mu \mathrm{m}$ required for advanced electrical test equipment.

In Section 2, a rigid body dynamics model of the moving platform subjected to an offset driving force is described. In Section 3, contributions from the various factors on 
platform yaw motion are analyzed. The position error due to the platform yaw is predicted and improved by a new $x-y$ stage design in Section 4 . The improved platform position repeatability with optimized centers of mass and stiffness is verified with a test bed in Section 5.

\section{Dynamics Model for Moving Platform}

The single axis stage has a pair of LM Guides jointed to a platform unit driven by a linear motor or a ball screw and motor assembly. In the following, the motion of platform is represented by a rigid body dynamics model.

\subsection{Yaw error Motion}

A single axis linear stage consists of a moving platform mounted on a pair of LM Guides. The platform is driven by a linear actuator as illustrated in Figure 2. The system may be characterized by center of mass of the platform, the center of stiffness of the LM Guides, and the driving force offset with respect to the geometric center of platform. In this system, the ratio of platform length $(l)$ in the motion direction to the width $(w)$ is known to be very important for drive stability. Typically, $l / w>1$ is required, and 1.6 or higher is recommended. [1]

When the driving force $(F)$ offset is significant, yaw motion of the platform is induced by its inertia, reaction forces $\left(R_{a}\right.$ to $\left.R_{d}\right)$, and friction forces $(\mu R a$ to $\mu R d$ ), leading to possible jamming.

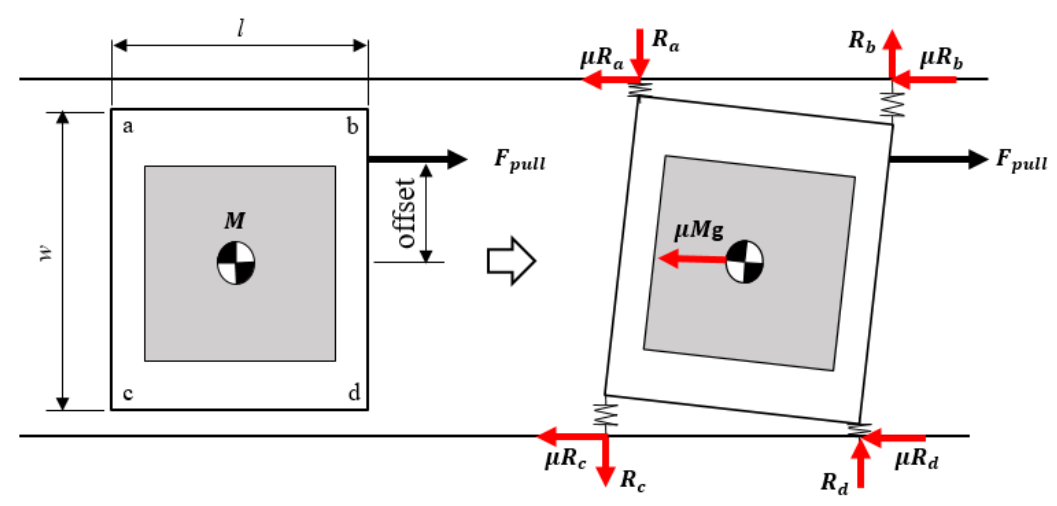

Figure 2. Schematic of an offset driven platform mounted on two linear motion guides

\subsection{Stiffness of LM Guide}

An LM Guide is composed of a rail, one or two blocks, and balls as shown in Figure 3(a). The balls rotate, enabling the block to perform a linear motion along the rail. The stiffness between the block and rail is analogous to a set of springs that resist external forces applied to the block in the horizontal and radial directions as shown in Figure 3(b). [3-15]

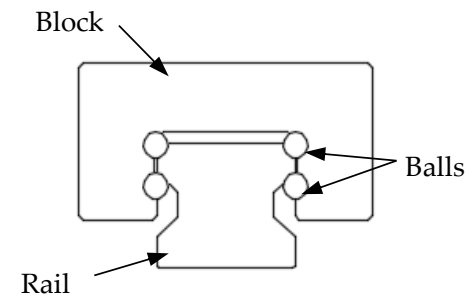

(a)

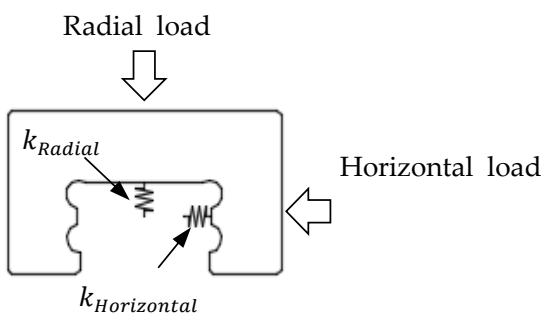

(b) 
Figure 3. LM Guide (a) componets and (b) stiffness elements.

Table 1. Stiffness of HSR LM Guides.

\begin{tabular}{|c|c|c|c|}
\hline & $\begin{array}{l}\text { Horizontal stiffness } \\
\qquad(\mathrm{N} / \mathrm{mm})\end{array}$ & $\begin{array}{l}\text { Radial stiffness } \\
\qquad(\mathrm{N} / \mathrm{mm})\end{array}$ & $\begin{array}{c}\text { Effective load } \\
(\mathrm{kN})\end{array}$ \\
\hline HSR20 & 249,938 & 348,602 & 1.4 \\
\hline HSR20L & 335,188 & 472,255 & 2.3 \\
\hline HSR25L & 334,806 & 488,448 & 2.8 \\
\hline HSR30L & 453,289 & 645,000 & 3.8 \\
\hline HSR35L & 495,000 & 681,385 & 5.5 \\
\hline
\end{tabular}

Table 1 shows the stiffness data of the LM Guides from THK's HSR models. The stiffness values were extracted from the catalog's experimental load vs. displacement graphs.

\subsection{Equations of Motion}

A free body diagram is shown in Figure 4 showing the reactions between the moving platform and two LM Guides. The platform guided by Rail \#1 and Rail \#2 is driven by force $(F)$ offset from the platform's center of geometry by distance $(d)$.

The Blocks of Rail $\# 1$ are numbered 1 and 2, respectively, while those of Rail $\# 2$ are numbered 3 and 4 , which are symmetric about the $y$ axis by distances of $l$ and $q$, respectively. The Blocks have horizontal stiffness of $k_{n}$ and a damping coefficient of $c_{n} .(n=1,2$, $3,4)$.

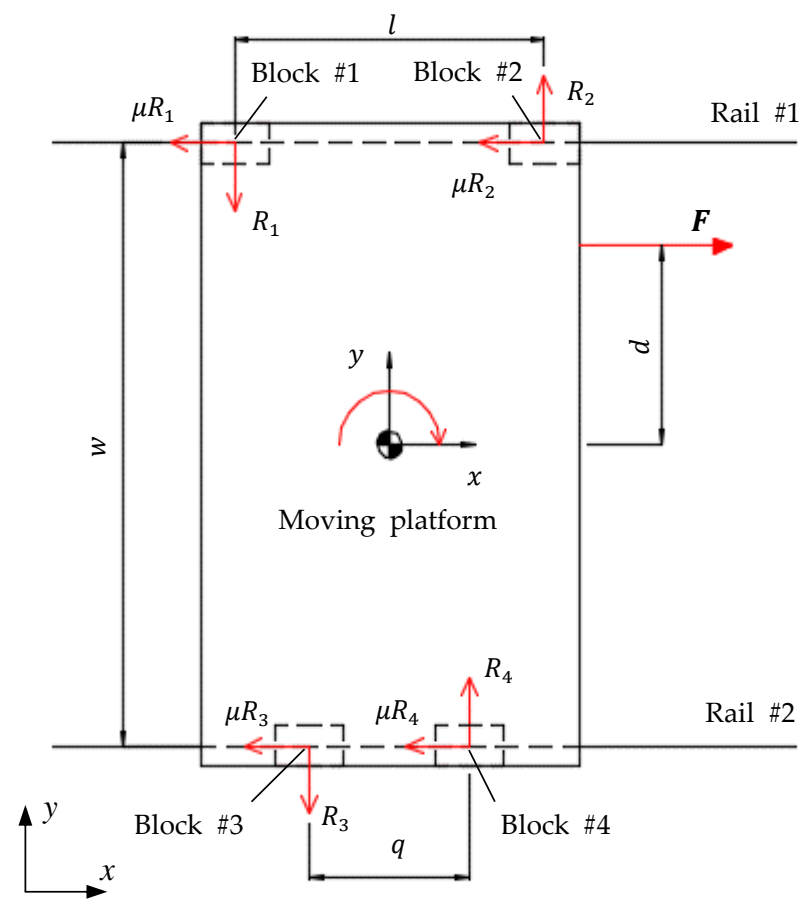

Figure 4. Free body diagram of the moving platform 
The Cartesian coordinate system represents the movement direction and the width direction as $x$ and $y$ and the direction perpendicular to the $x-y$ plane as $z$.

Figure 5. shows a schematic diagram of the platform rotated by angle of $\theta$ by the driving force $F$. The coordinates $\left(x_{c}, y_{c}\right)$ and $\left(x_{p}, y_{p}\right)$ refer to the center of mass and the center of rotation respectively.

$$
\begin{aligned}
& \sum F_{x}=m \ddot{x}_{p} \\
& \sum F_{y}=m \ddot{y}_{p} \\
& \sum M=I \ddot{\theta}
\end{aligned}
$$

The force equilibrium equation for platform is given as Equation (1), and the rotational inertia is given as Equation (2).

$$
I=m\left(\frac{w^{2}+l^{2}}{12}+\left(x_{p}-x_{c}\right)^{2}+\left(y_{p}-y_{c}\right)^{2}\right)
$$

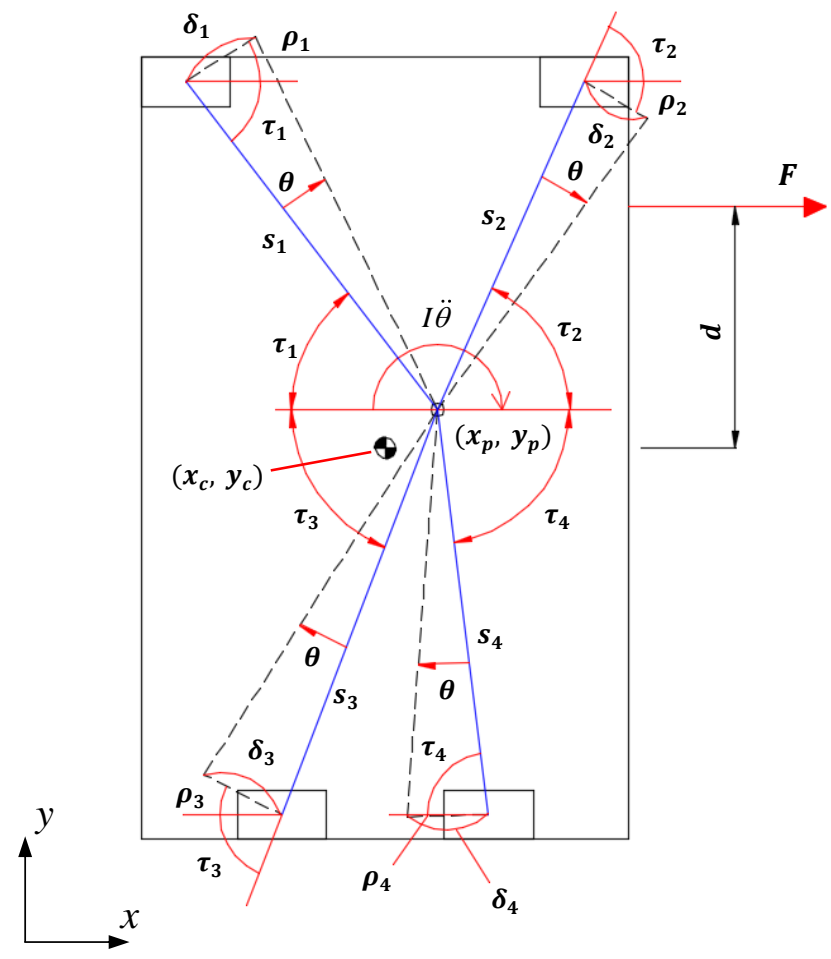

Figure 5. Schematic diagram of yaw motion.

The position of the rotational center (Pole) is a function of time, and the lengths of lines $\left(s_{n}\right)$ from the pole to the block centers are given as,

$$
\begin{aligned}
& s_{1}(t)=\sqrt{\left(l / 2+x_{p}(t)\right)^{2}+\left(w / 2-y_{p}(t)\right)^{2}} \\
& s_{2}(t)=\sqrt{\left(l / 2-x_{p}(t)\right)^{2}+\left(w / 2-y_{p}(t)\right)^{2}} \\
& s_{3}(t)=\sqrt{\left(q / 2+x_{p}(t)\right)^{2}+\left(w / 2+y_{p}(t)\right)^{2}} \\
& s_{4}(t)=\sqrt{\left(q / 2-x_{p}(t)\right)^{2}+\left(w / 2+y_{p}(t)\right)^{2}}
\end{aligned}
$$


while the angles $\left(\tau_{n}\right)$ between the lines $s_{n}$ and the $x$ axis is given as,

$$
\begin{aligned}
& \tau_{1}(t)=\tan ^{-1}\left(\frac{w / 2-y_{p}(t)}{x_{p}(t)+l / 2}\right) \\
& \tau_{2}(t)=\tan ^{-1}\left(\frac{w / 2-y_{p}(t)}{-x_{p}(t)+l / 2}\right) \\
& \tau_{3}(t)=\tan ^{-1}\left(\frac{w / 2+y_{p}(t)}{x_{p}(t)+q / 2}\right) \\
& \tau_{4}(t)=\tan ^{-1}\left(\frac{w / 2+y_{p}(t)}{-x_{p}(t)+q / 2}\right)
\end{aligned}
$$

The angle $\rho_{n}$ between the block center displacement vectors $\delta_{n}$ and the $x$ axis can be derived as shown in Equation (5).

$$
\begin{aligned}
& \rho_{1}(t)=-\frac{\theta(t)+2 \tau_{1}(t)}{2}+\frac{\pi}{2} \\
& \rho_{2}(t)=\frac{\theta(t)-2 \tau_{2}(t)}{2}+\frac{\pi}{2} \\
& \rho_{3}(t)=\frac{\theta(t)-2 \tau_{3}(t)}{2}+\frac{\pi}{2} \\
& \rho_{4}(t)=-\frac{\theta(t)+2 \tau_{4}(t)}{2}+\frac{\pi}{2}
\end{aligned}
$$

The displacement vectors $\delta_{n}$ are decomposed into to $\delta_{n x}$ and $\delta_{n y}$, as shown in Figure 6 . The displacements in the $y$ direction and their time rate of changes are given as,

$$
\begin{aligned}
& \delta_{\mathrm{ny}}(\mathrm{t})=\mathrm{s}_{\mathrm{n}}(\mathrm{t}) \theta(\mathrm{t}) \sin \rho_{\mathrm{n}}(\mathrm{t}) \\
& \dot{\delta}_{\mathrm{ny}}(\mathrm{t})=\dot{\mathrm{s}}_{\mathrm{n}} \theta \sin \rho_{\mathrm{n}}+\mathrm{s}_{\mathrm{n}}(\mathrm{t}) \dot{\theta}(\mathrm{t}) \sin \rho_{\mathrm{n}}(\mathrm{t})+\mathrm{s}_{\mathrm{n}}(\mathrm{t}) \dot{\rho}_{\mathrm{n}}(\mathrm{t}) \cos \rho_{\mathrm{n}}(\mathrm{t})
\end{aligned}
$$

The horizontal reaction forces $R_{n}$ on the blocks are functions of the displacements $\delta_{n y}$ given as

$$
R_{n}=k_{n} \delta_{n y}+c_{n} \dot{\delta}_{n y} \quad \text { where } \mathrm{n}=1,2,3,4
$$

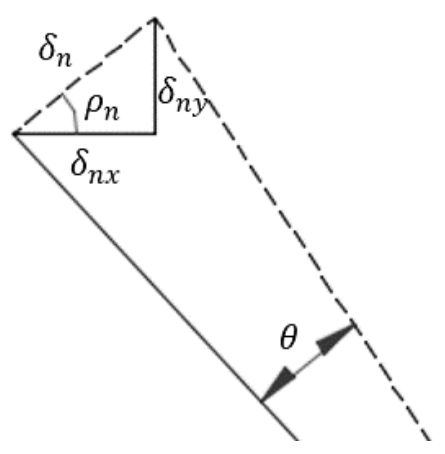

Figure 6. Vector decomposition of rotation block displacement 
The relation between the center of mass $\left(x_{c}, y_{c}\right)$ and the center of rotation $\left(x_{p}, y_{p}\right)$ is given as following where $y_{p}(0)$ refers to the initial $\mathrm{y}$-coordinate value of the platform's center of mass.

$x_{c}=\frac{y_{c}(0)}{w / 2}\left(\frac{-s_{1} \cos \left(\tau_{1}+\theta\right)+s_{2} \cos \left(\tau_{2}-\theta\right)+s_{3} \cos \left(\tau_{3}-\theta\right)-s_{4} \cos \left(\tau_{4}+\theta\right)}{4}\right)+\frac{4 x_{p}-s_{1} \cos \left(\tau_{1}+\theta\right)+s_{2} \cos \left(\tau_{2}-\theta\right)-s_{3} \cos \left(\tau_{3}-\theta\right)+s_{4} \cos \left(\tau_{4}+\theta\right)}{4}$
$y_{c}=\frac{y_{c}(0)}{w / 2}\left(\frac{s_{1} \sin \left(\tau_{1}+\theta\right)+s_{2} \sin \left(\tau_{2}-\theta\right)+s_{3} \sin \left(\tau_{3}-\theta\right)+s_{4} \sin \left(\tau_{4}+\theta\right)}{4}\right)+\frac{4 y_{p}+s_{1} \sin \left(\tau_{1}+\theta\right)+s_{2} \sin \left(\tau_{2}-\theta\right)-s_{3} \sin \left(\tau_{3}-\theta\right)-s_{4} \sin \left(\tau_{4}+\theta\right)}{4}$

Substituting Equations (2) to (8) into the force equilibrium Equation (1) of the free body diagram shown in Figure 4 leads to the following equations of yaw motion.

$$
\begin{aligned}
& \sum F_{x}: F-\mu m g-m\left(R_{1}+R_{2}+R_{3}+R_{4}\right)=m \ddot{x}_{p} \\
& \sum F_{y}:-R_{1}+R_{2}-R_{3}+R_{4}-\mu m g=m \ddot{y}_{p} \\
& \sum M: F\left(d-y_{p}\right)-\left(x_{p}-\mu y_{p}+\frac{l+\mu w}{2}\right) R_{1}+\left(x_{p}+\mu y_{p}-\frac{l+\mu w}{2}\right) R_{2}-\left(x_{p}-\mu y_{p}+\frac{q-\mu w}{2}\right) R_{3}+\left(x_{p}+\mu y_{p}-\frac{q-\mu w}{2}\right) R_{4}=I \ddot{\theta}
\end{aligned}
$$

\section{Factors Affecting the Yaw Motion}

The yaw motion of the moving platform depends on the platform aspect ratio, platform's center of mass position, driving force offset ratio, parallelism between the two LM Guides, and the LM Guides' center of stiffness position. [1, 18, 19]

These factors can be classified into controllable and uncontrollable parameters depending on the situation. In this work, platform's aspect ratio and driving force offset were uncontrollable under the given conditions, while the parallelism between the LM guides, the center of mass, and the center of stiffness were defined as controllable parameters for design and implementation.

The yaw motion of the moving platform may be described by yaw angle $(\theta)$ and settling time $(\lambda)$, respectively, as functions of parallelism $(P A)$, the center of mass $(C M)$, the center of stiffness (CS), and force offset $(F O)$.

$$
\begin{aligned}
& \text { Yaw motion }=f(\theta, \lambda) \\
& \theta=\left.\theta(P A, C S, C M)\right|_{F O} \\
& \lambda=\left.\lambda(P A, C S, C M)\right|_{F O}
\end{aligned}
$$

The yaw angle can be predicted for given size of the platform, the installation positions of the LM Guides, and the driving force from Equation (10).

Numerical solutions to Equation (10) were obtained using the GEKKO package of python as a solver. GEKKO is a package for machine learning and the optimization of mixed-integer and differential algebraic equations. [24]

\subsection{Uncontrollable Prameters: Given Conditions}

\subsubsection{Aspect Ratio of Platforms}

The effects of platform aspect ratio and force offset has been investigated as shown in Table 2 and Figure 7. Four platforms with different sizes were selected. The length $(l)$ in the moving direction was fixed at $0.5 \mathrm{~m}$, and the width $(w)$ was varied from $0.3-1.0 \mathrm{~m}$, resulting in the $l / w$ ratio of 1.7 to 0.5 . LM Guide model HSR20 was selected with blocks on the four corners of the platform. The driving motion profile is trapezoidal with an acceleration of $1 \mathrm{~g}\left(9.8 \mathrm{~m} / \mathrm{s}^{2}\right)$ and an acceleration time of $0.1 \mathrm{~s}$. 
Table 2. Dimensions of Moving platform.

\begin{tabular}{ccccc}
\hline \hline No. & $\begin{array}{c}\text { Length, } l \\
(\mathbf{m})\end{array}$ & $\begin{array}{c}\text { Width, } w \\
(\mathbf{m})\end{array}$ & $\begin{array}{c}\text { Weight } \\
(\mathbf{k g})\end{array}$ & $\boldsymbol{l} / \boldsymbol{w}$ \\
\hline 1 & 0.5 & 0.3 & 29.3 & 1.7 \\
2 & 0.5 & 0.5 & 48.8 & 1.0 \\
3 & 0.5 & 0.8 & 78.0 & 0.6 \\
4 & 0.5 & 1.0 & 97.5 & 0.5 \\
\hline \hline
\end{tabular}

No.1

No.2

No.3

No.4

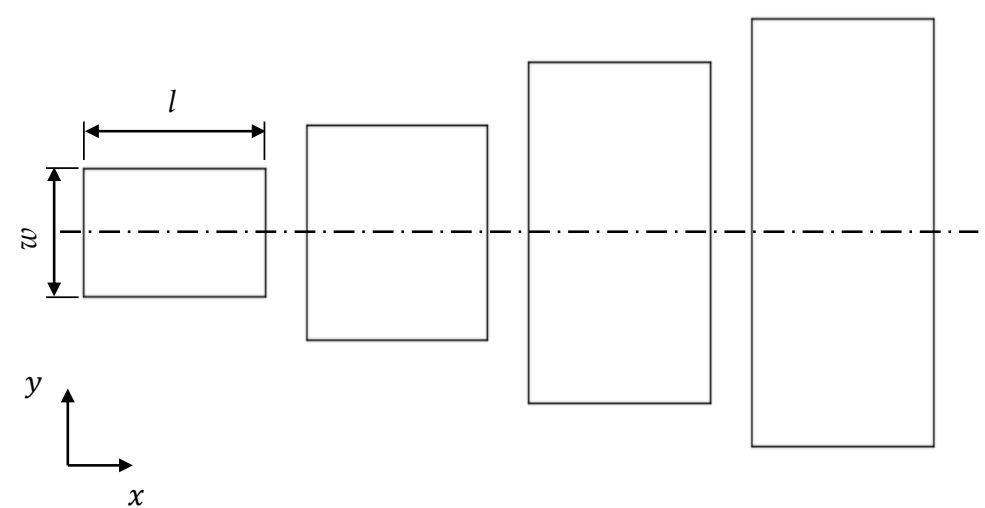

Figure 7. Comparison of Moving Platforms

The friction force and damping coefficient of LM Guides vary depending on the operating environment, maintenance, and driving conditions. [21, 22] In this work, friction coefficient $(f)$ and the damping coefficients $(c)$ were fixed at 0.02 and $10 \mathrm{Nsec} / \mathrm{mm}$, respectively.

\subsubsection{Force Offset}

The force offset $(F O)$ may be defined in terms of the distance between the ball screw linear actuator illustrated in Figure 1 and the geometric center of the moving platform. In its normalized form the force offset is defined as

$$
\text { FO }(\text { Force Offset })=\frac{d}{w / 2} \times 100 \quad(\%)
$$

where $d$ is the offset distance and $w$ is the width of the platform. Figure 8 illustrates the parameters for $F O$ and examples of different $F O$ configurations. 


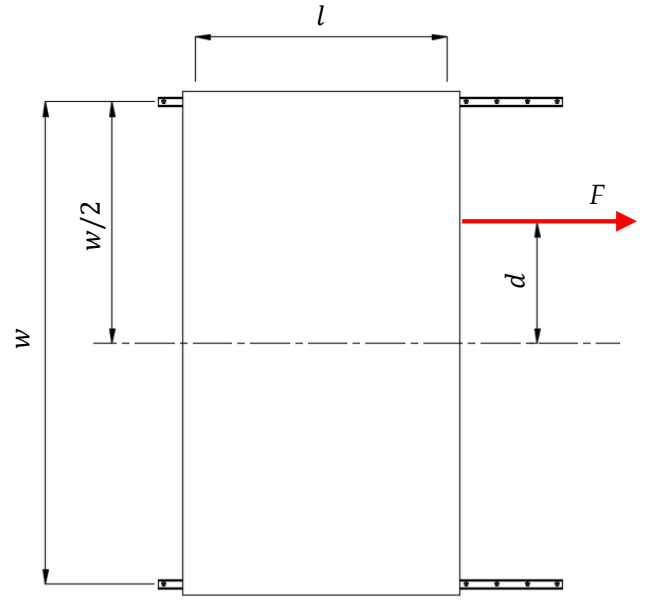

(a)
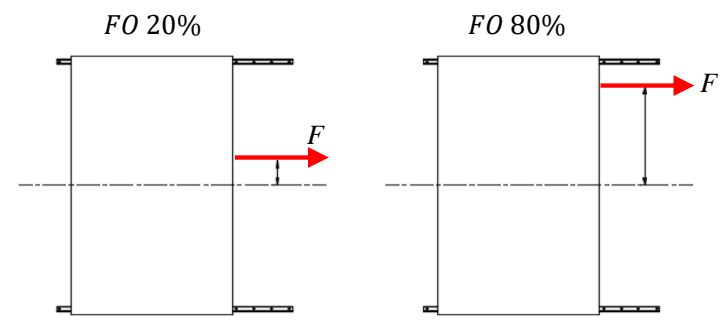

(b)

Figure 8. (a) Schematic diagram of $F O$ definition and (b) comparison of $F O 20 \%$ \& $F O 80 \%$

Figure 9 shows yaw vibration of the No. 4 platform (Figure 7) driven with four different values of FOs. The vibration response of the moving platform in Figure 9 is typical for the trapezoidal motion profile. [23] The yaw angle is observed to converge after a certain period of time, also indicating that the higher the $F O$, the higher the convergence value becomes.

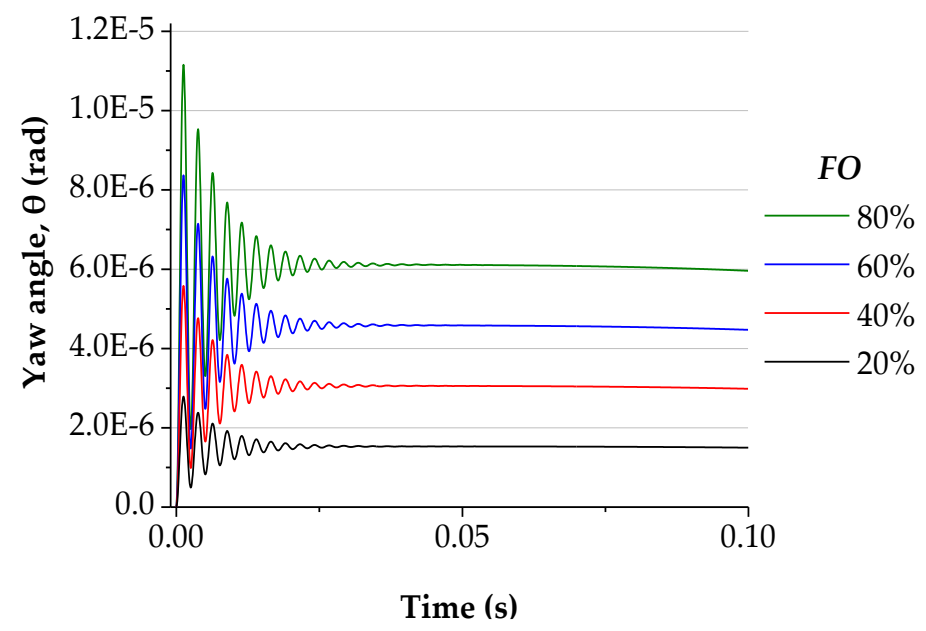

Figure 9. Yaw angle in respect to driving forece position (No. 4) 


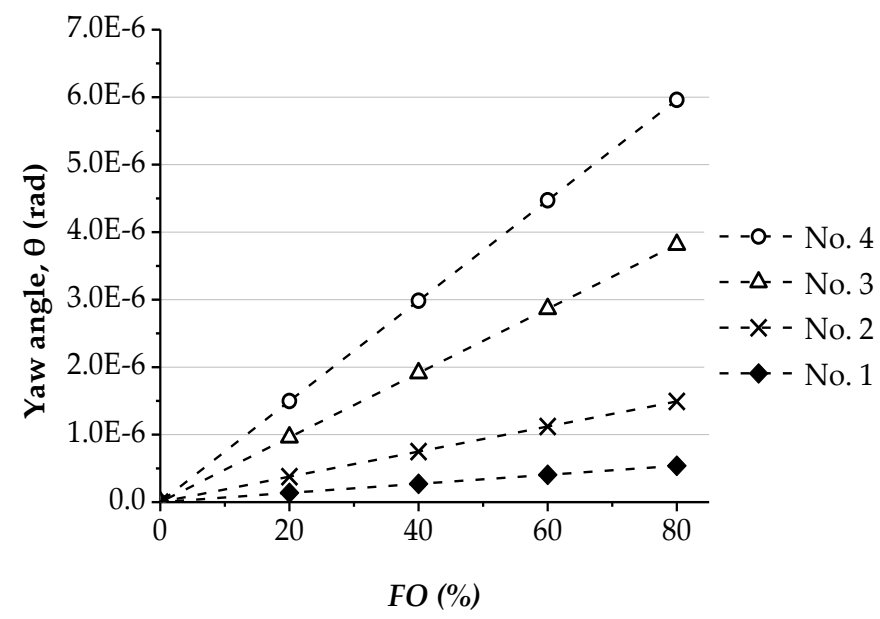

Figure 10. Yaw angle in repecto to $F O$

Figure 10 presents the convergence value of yaw angle vs. $F O$ value for the four platform types, which shows that the larger the $l / w$ value, the smaller the $F O$ effect on the yaw angle.

Figure 11 is an enlarged graph of FO $80 \%$ in Figure 9. The settling time $(\lambda)$ is when the vibration value becomes less than the error band, $0.1 \mathrm{E}-6 \mathrm{rad}$, and it is about $33 \mathrm{~ms}$.

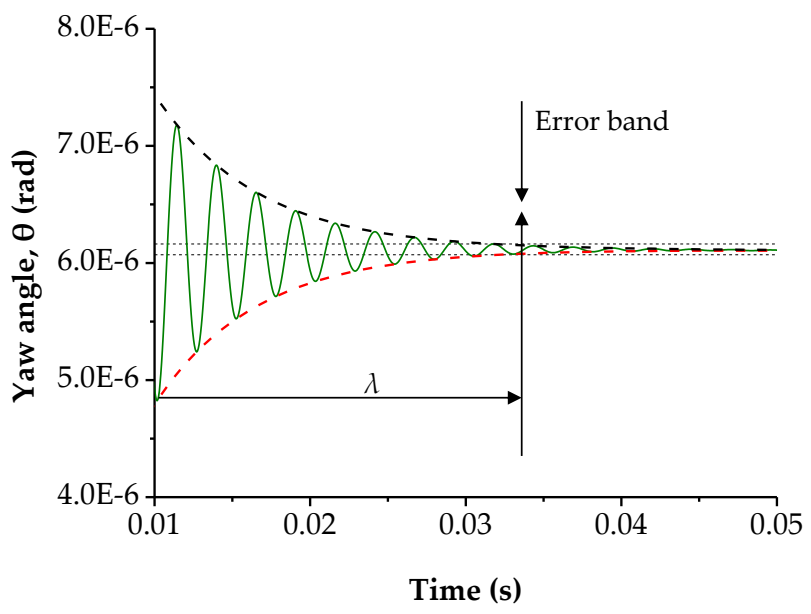

Figure 11. Zoomed yaw angle graph: Settling time $(\lambda)$ and Error bank.

\subsubsection{Verification of Dynamics Model}

The predictions with Equation (10) were compared with the finite element analysis results obtained with ANSYS, a commercial finite element analysis (FEA) tool. In the ANSYS FEA model, the balls between the block and rail of the LM Guide were represented by bushing joint which requires two opposing contact surfaces which are described as reference and mobile in Figure 12(a). The constraint of a bushing joint was applied with radial and horizontal stiffness values (Figure 3 ) the mobile and reference surfaces. Figure 12(b) shows the finite element mesh for FEA.

No. 4 platform in Table 2 was selected for the simulation model and the analysis was performed in the $20 \%-80 \%$ range of $F O$ values. 


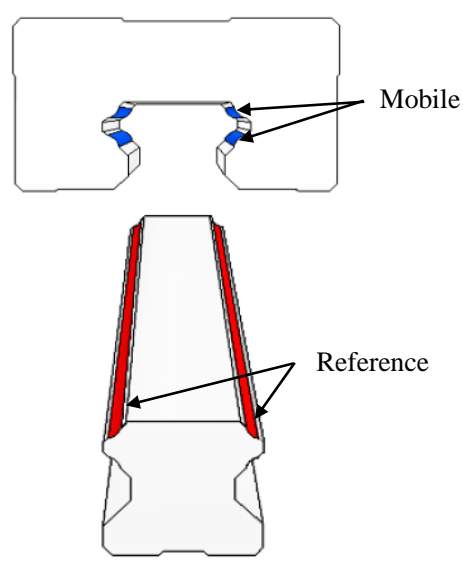

(a)

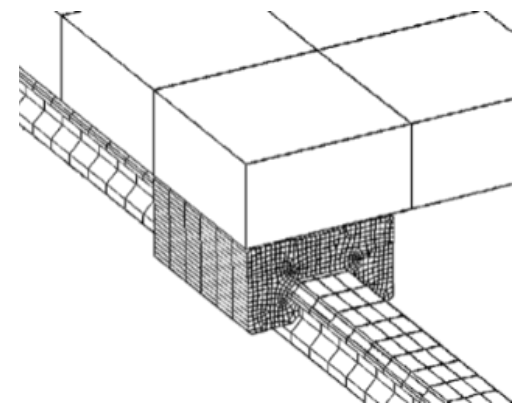

(b)

Figure 12. Finite element model for LM Guide: (a) contact surfaces of a bushing joint, and (b) finite element mesh.

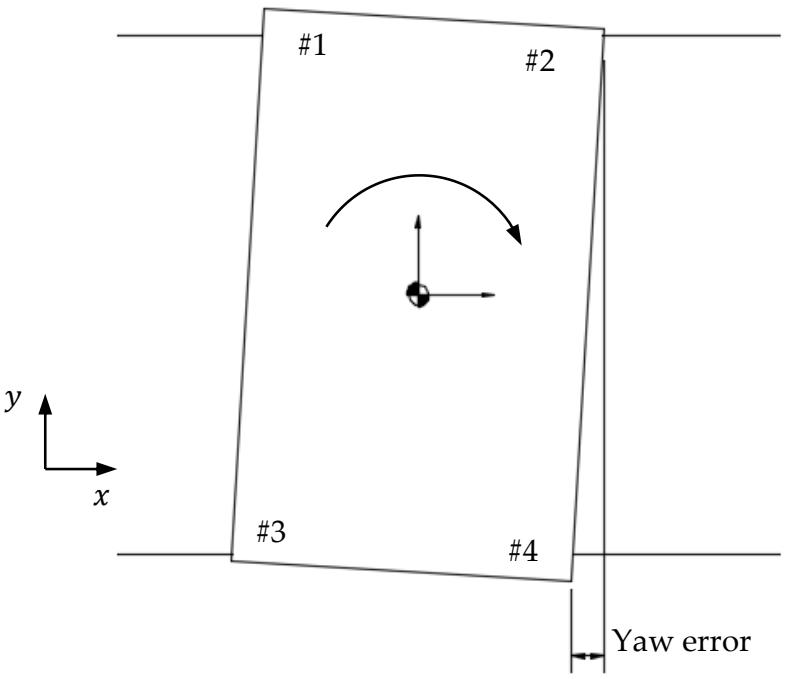

Figure 13. Schematic of yaw error.

The yaw error of the stage is represented by difference between the $x$-axis coordinate difference of block 2 and block 4 as illustrated in Figure 13. The yaw error values from the FEM simulations as well as from Equation (10) are listed in Table 3. With FO, the yaw error tended to increase in both results of the simulations and the dynamics Equation (10).

Table 3. Yaw error predictions for the No. 4 platform, $(\mu \mathrm{m})$

\begin{tabular}{ccc}
\hline \hline FO & $\begin{array}{c}\text { FEM simulation } \\
\text { ANSYS }\end{array}$ & $\begin{array}{c}\text { Rigid dynamics model } \\
\text { Equation (10) }\end{array}$ \\
\hline $0 \%$ & 0 & 0 \\
$20 \%$ & 0 & 2 \\
$40 \%$ & 3 & 3 \\
$60 \%$ & 6 & 4 \\
$80 \%$ & 7 & 6 \\
\hline \hline
\end{tabular}


The difference between the predictions from FEM model and rigid body dynamics model may be due to the structural vibration ignored in the rigid body dynamics model. It may also be due to the difference between the bushing joint model and simple spring model for the LM Guide stiffness. Even with these differences, simpler and faster rigid body dynamics model may be a useful tool for the predictions of yaw error at the early stage of precision linear stage design.

\subsection{Controllable Prameters: Design, Manufacturing Conditions}

\subsubsection{Parallelism between LM Guides}

Ideally, a pair of LM Guide rails should be perfectly parallel. In reality, this cannot be the case due to the assembly error of LM Guides in addition to the machining error of the installation surface itself.

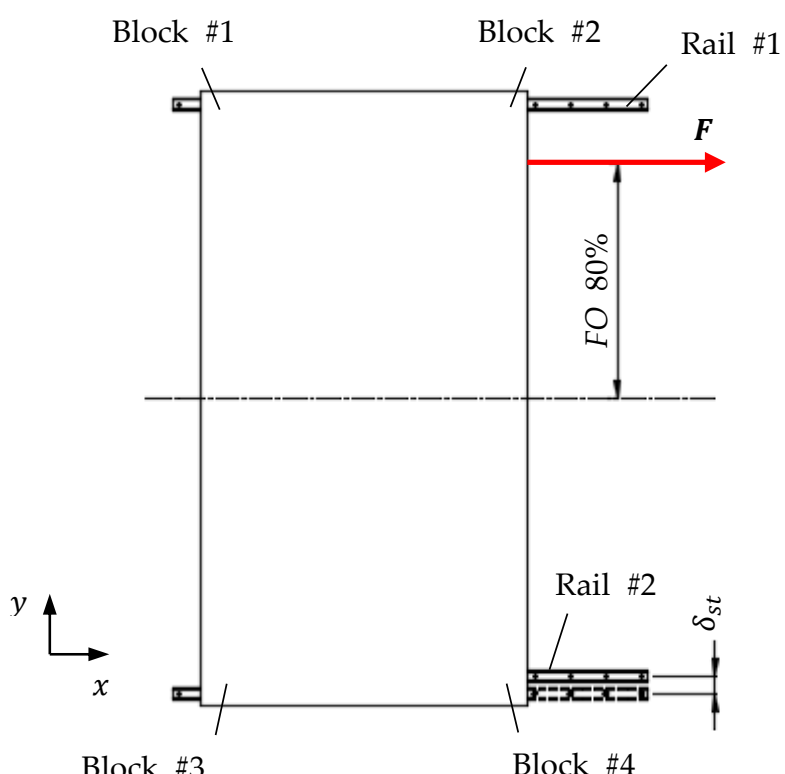

Figure 14. Parallelism error between LM Guides

As shown in Figure 14, LM Rail \#1 is an ideal straight line, and Rail \#2 represents the straightness error in the $y$-direction by $\delta_{s t}$ from the Block \#4 position. This means an application of an external force of $\delta_{s t} \times k_{4}$ to the \#4 location in the $y$ direction. The yaw angle was calculated by reflecting the additional external force in the yaw motion Equation (10). 


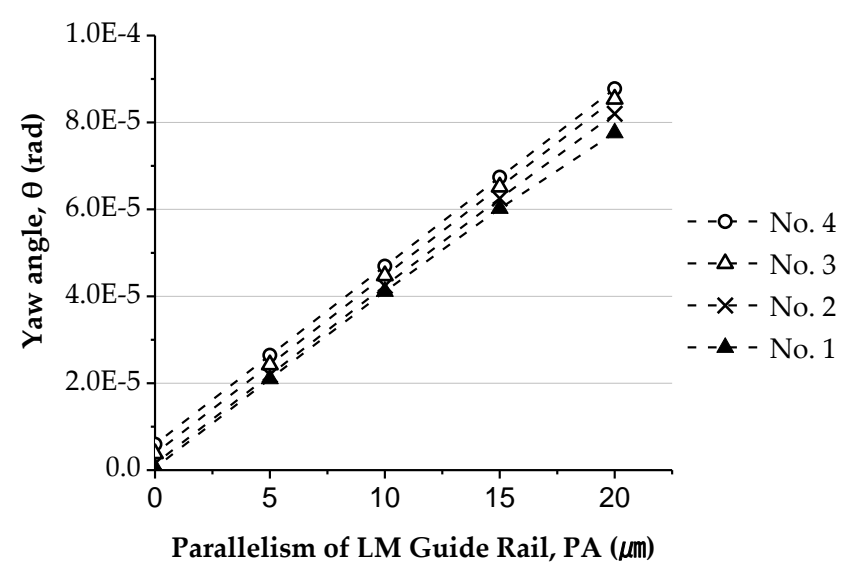

Figure 15. Results of the yaw angle of platforms.

The driving force was applied to the four types of platforms at $F O$ of $80 \%$ with the trapezoidal motion profile mentioned earlier. The convergence values of the yaw angle according to the parallelism error are shown in Figure 15. The yaw angle at 0 parallelism is equivalent to the value of $F O 80 \%$ in Figure 10. With parallelism, the yaw angle is increased by several times or more regardless of $l / w$. Parallelism is the most dominant factor affecting the yaw motion regardless of $l / w$ ratio or $F O$.

\subsubsection{Center of mass \& Center of stiffness}

The moving platform has several midpoints: centers of geometry, mass, and stiffness. These midpoints are defined as

Center of geometry $=O(0,0)$

Center of mass $=O^{\prime}\left(\frac{\int x d m}{m}, \frac{\int y d m}{m}\right)$

Center of stiffness $=O^{\prime \prime}\left(\frac{\sum x_{i} k_{i}}{\sum k_{i}}, \frac{\sum y_{i} k_{i}}{\sum k_{i}}\right)$

The midpoints are illustrated in Figure 16. 


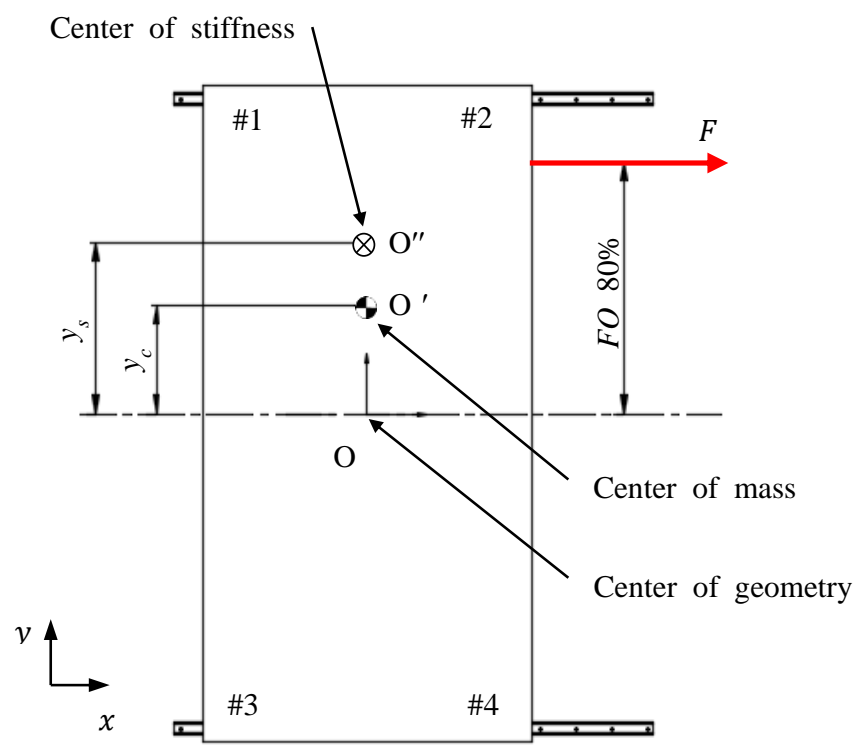

Figure 16. Midpoints of the platform

Ratio of center of mass (CM) and ratio of center of stiffness (CS) are defined as the ratios of the distances from the center of geometry (origin $\mathrm{O}$ ) to each center point in the width direction to the half-width of the platform as

$$
\begin{aligned}
& C M(\text { Ratio of Center of Mass })=\frac{y_{c}}{w / 2} \times 100(\%) \\
& C S(\text { Ratio of Center of Stiffness })=\frac{y_{s}}{w / 2} \times 100(\%)
\end{aligned}
$$

The center of mass can be modified by adding or removing weights for the given platform size. The center of stiffness can be modified by changing the positions of the LM Guides, installing additional guides, or replacing the LM Guide types.

In general, two blocks on a LM guide rail are identical and thus $k_{1}=k_{2}, k_{3}=k_{4}$. Table 4 lists some $C S$ values for the $k_{3} / k_{1}$ ratios.

Table 4. Ratio of center of stiffness $C S$ for $k_{3} / k_{1}$ values

\begin{tabular}{ccccccc}
\hline \hline $\mathrm{CS}$ & $\mathbf{0 \%}$ & $\mathbf{2 0 \%}$ & $\mathbf{4 0 \%}$ & $\mathbf{6 0 \%}$ & $\mathbf{8 0 \%}$ & $\mathbf{1 0 0 \%}$ \\
\hline $\mathrm{k}_{3} / \mathrm{k}_{1}$ & 1 & 0.7 & 0.4 & 0.30 & 0.1 & 0 \\
\hline \hline
\end{tabular}

The yaw motion dependence on CS and CM for No. 4 platform with zero parallelism at $80 \%$ of $F O$ such as

$$
\begin{aligned}
& \theta=\left.\theta(P A: 0, C S, C M)\right|_{F O: 80 \%, \text { No 4Platform }} \\
& \lambda=\left.\lambda(P A: 0, C S, C M)\right|_{F O: 80 \%, \text { No4Platform }}
\end{aligned}
$$

is described in Figure 17 and Figure 18. 


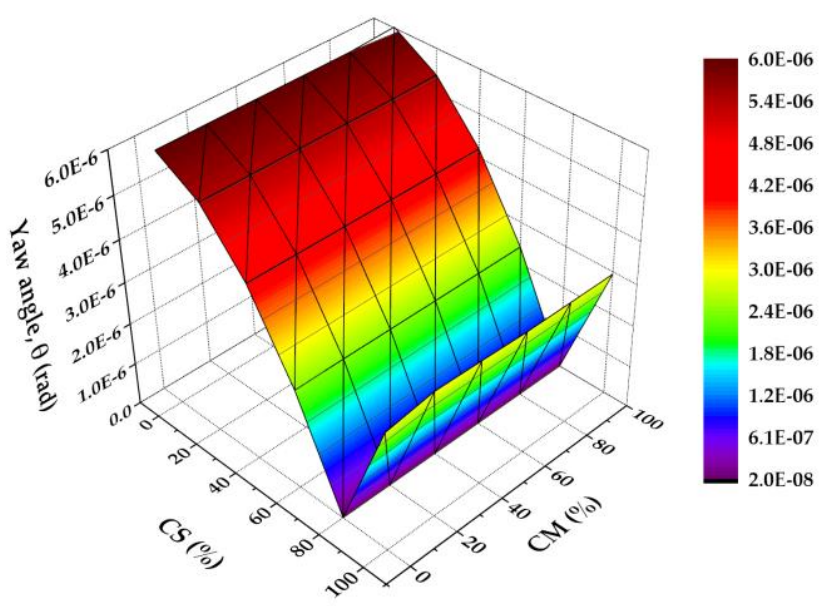

Figure 17. Convergence yaw angle $(\theta)$ vs. $C M$ and $C S$.

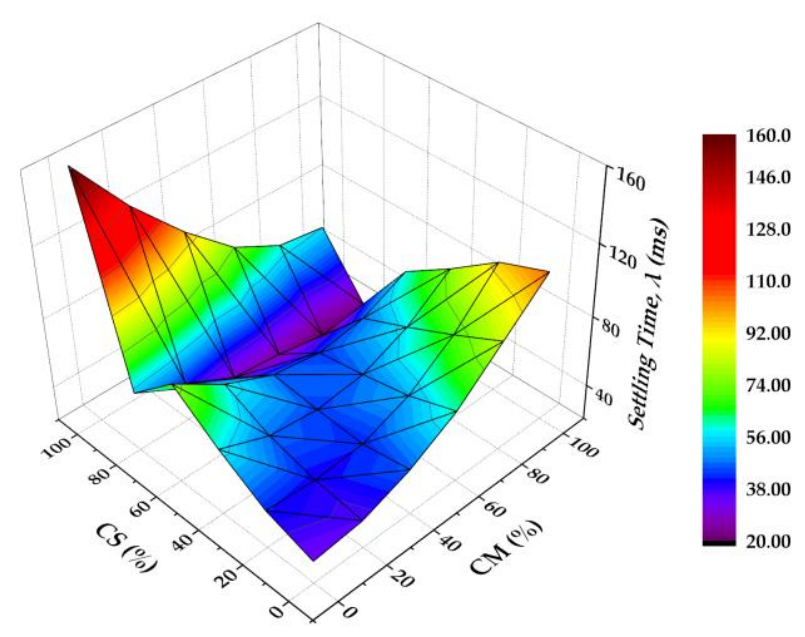

Figure 18. Settling time $(\lambda)$ vs. $C M$ and $C S$.

Figure 17 shows the absolute value of the yaw angle for a range of $C S$ and $C M$, and Figure 18 shows the settling time. The yaw angle is greatly affected by CS while the effect of $C M$ is minimal. The yaw angle is smallest at CS of $80 \%$ for $F O$ of $80 \%$. Figure 18 shows that the settling time is also minimal when $C S$ is same as $C M$. Lowest settling time is also achieved at CS of $80 \%$.

As for the yaw angle, Figure 17 indicates that it is governed primarily by CS indicating that the LM Guides are the most dominant elements constraining the yaw rotation of platform. For lower range of $C S$ values, the centers of stiffness and rotation are relatively close to each other. As CS increases closer to the value of $F O$, the constrains from the guide rail $\# 2$ are partially reduced and thus the center of rotation also moves closer to $F O$ position. This will reduce the yaw moment by the driving force $F$ about the center of rotation. Also the rotational inertia of the platform is increased since the rotation center moves away from the center of mass.

Settling time is a result of complex interplay among $C S, C M$ and $F O$ as indicated in Figure 18. It turns out that the settling time reaches its minimum at the values of $C S$ and $C M$ similar to $F O$ of $80 \%$. In this case, the centers of rotation, mass and stiffness are closer to the $F O$ position. This will reduce the yaw moment as well as rotational inertia.

The data for Figure 17and Figure 18 are listed in Table 5 and Table 6. 


\begin{tabular}{|c|c|c|c|c|c|c|}
\hline $\mathrm{CM}$ & $0 \%$ & $20 \%$ & $40 \%$ & $60 \%$ & $80 \%$ & $100 \%$ \\
\hline $0 \%$ & 5.96 & 5.38 & 4.19 & 2.39 & 0.0012 & 2.83 \\
\hline $20 \%$ & 5.96 & 5.38 & 4.19 & 2.40 & 0.0018 & 3.01 \\
\hline $40 \%$ & 5.96 & 5.38 & 4.19 & 2.40 & 0.0021 & 2.98 \\
\hline $60 \%$ & 5.96 & 5.38 & 4.19 & 2.40 & 0.0020 & 2.94 \\
\hline $80 \%$ & 5.96 & 5.38 & 4.19 & 2.40 & 0.0020 & 2.94 \\
\hline $100 \%$ & -6.02 & 5.40 & 4.20 & 2.40 & 0.0020 & 2.94 \\
\hline
\end{tabular}

Table 6. Settling time for combinations of CM and CS. (ms)

\begin{tabular}{rrrrrrr}
\hline \multicolumn{1}{c}{ CS } & $\mathbf{0 \%}$ & $\mathbf{2 0 \%}$ & $\mathbf{4 0 \%}$ & $\mathbf{6 0} \%$ & $\mathbf{8 0} \%$ & $\mathbf{1 0 0 \%}$ \\
\hline $0 \%$ & 33 & 42 & 58 & 77 & 54 & 162 \\
$20 \%$ & 36 & 40 & 47 & 59 & 42 & 127 \\
$40 \%$ & 46 & 42 & 44 & 47 & 28 & 98 \\
$60 \%$ & 61 & 52 & 47 & 43 & 25 & 74 \\
$80 \%$ & 83 & 70 & 58 & 47 & 22 & 60 \\
$100 \%$ & 105 & 95 & 76 & 59 & 23 & 56 \\
\hline \hline
\end{tabular}

For the conditions where $C S$ and $C M$ have an identical value, the effects of parallelism $P A$ on yaw angle and settling time such as

$$
\begin{aligned}
& \theta=\left.\theta(P A, C S=C M)\right|_{F O 80 \%, \text { No4 Platform }} \\
& \lambda=\left.\lambda(P A, C S=C M)\right|_{F O 80 \%, \text { No4Plafform }}
\end{aligned}
$$

may be investigated.

Perfect parallelism between two LM Guide rails is impossible. The practical lower limit for $P A$ is $10 \mu \mathrm{m}$ for the moving platform. The maximum admissible settling time is $100 \mathrm{~ms}$ for the current motion profile of $1 \mathrm{~g}$ and 0.1 second for acceleration and deceleration.

Figure 19 suggests that when the value of $C S$ and $C M$ is maintained close to $F O$ of $80 \%$, allowable yaw angle is achieved for $P A$ of $10 \mu \mathrm{m}$. Figure 20 shows that settling time is maintained below $70 \mathrm{~ms}$ for the entire range of $C S$ and $C M$ and for $P A$ of $20 \mathrm{~mm}$ and beyond. The data for Figure 19 and Figure 20 are listed in Table 7 and Table 8. 


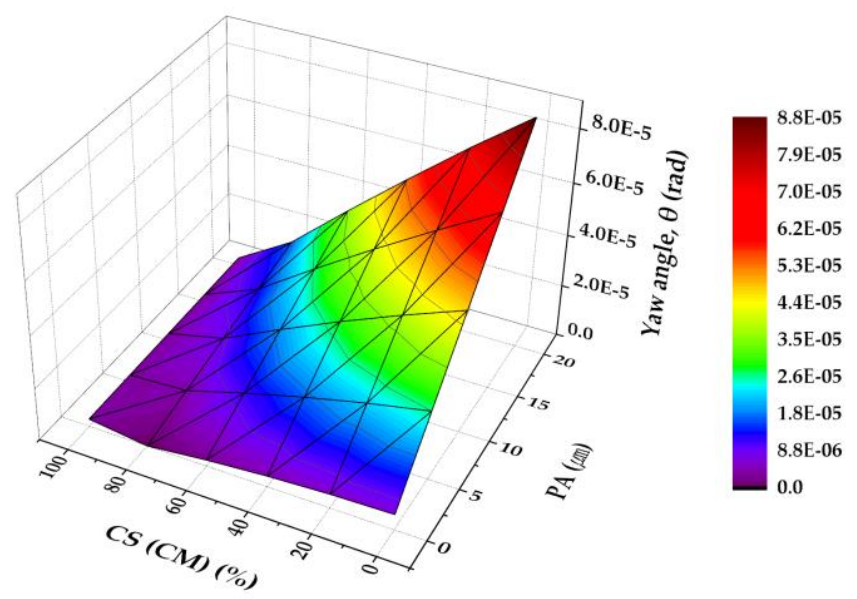

Figure 19. Yaw angle vs. $C S$ and $P A$

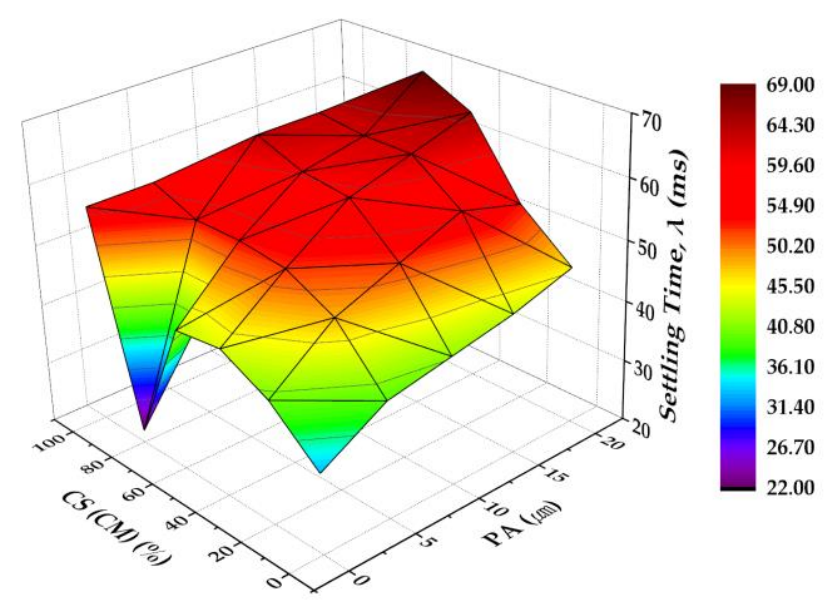

Figure 20. Settling time $(\lambda)$ vs. $C S$ and $P A$

Table 7. Convergence yaw angle for combinations of $P A$ and $C S$

\begin{tabular}{rlcccc}
\hline \hline PA & $\mathbf{0 \mu m}$ & $\mathbf{5 \mu \mathrm { m }}$ & $\mathbf{1 0 \mu \mathrm { m }}$ & $\mathbf{1 5} \boldsymbol{\mu m}$ & $\mathbf{2 0} \boldsymbol{\mu m}$ \\
\hline $0 \%$ & 5.96 & 26.4 & 46.9 & 67.4 & 87.8 \\
$20 \%$ & 5.38 & 21.8 & 38.2 & 54.6 & 71.1 \\
$40 \%$ & 4.19 & 16.5 & 28.8 & 41.1 & 53.4 \\
$60 \%$ & 2.40 & 10.6 & 18.7 & 26.9 & 35.1 \\
$80 \%$ & 0.002 & 4.04 & 8.08 & 12.1 & 16.2 \\
$100 \%$ & 2.92 & 2.96 & 3.00 & 3.04 & 3.08 \\
\hline \hline
\end{tabular}


Table 8. Settling time for combinations of $P A$ and $C S$

\begin{tabular}{rrrrrr}
\hline \hline PA & $\mathbf{0 \mu \mathrm { m }}$ & $\mathbf{5 \mu \mathrm { m }}$ & $\mathbf{1 0} \boldsymbol{\mu \mathrm { m }}$ & $\mathbf{1 5} \boldsymbol{\mu \mathrm { m }}$ & $\mathbf{2 0} \boldsymbol{\mu \mathrm { m }}$ \\
\hline $0 \%$ & 33 & 39 & 41 & 43 & 46 \\
$20 \%$ & 40 & 48 & 52 & 56 & 53 \\
$40 \%$ & 44 & 52 & 59 & 62 & 65 \\
$60 \%$ & 43 & 53 & 60 & 62 & 69 \\
$80 \%$ & 22 & 53 & 63 & 63 & 63 \\
$100 \%$ & 56 & 56 & 56 & 56 & 56 \\
\hline \hline
\end{tabular}

At this point, it may be suggested that the values of $C S, C M$ need to be close to the $F O$ of $80 \%$ while $P A$ may be maintained in the vicinity $10 \mu \mathrm{m}$. This will require the ratio $k_{3} / k_{1}$ less than 0.1 and balancing weight to the platform for $80 \%$ of $C M$. In practice, it is difficult to accurately match the ratio $k_{3} / k_{1}$ with commercially available LM Guides. One alternative is to choose $k_{3}=0$ with CS of $100 \%$. Keeping the blocks of rail \#2 simply supported in the $z$-direction without constraints in the $x$ and $y$ directions can be a practical solution as illustrated in Figure 21.

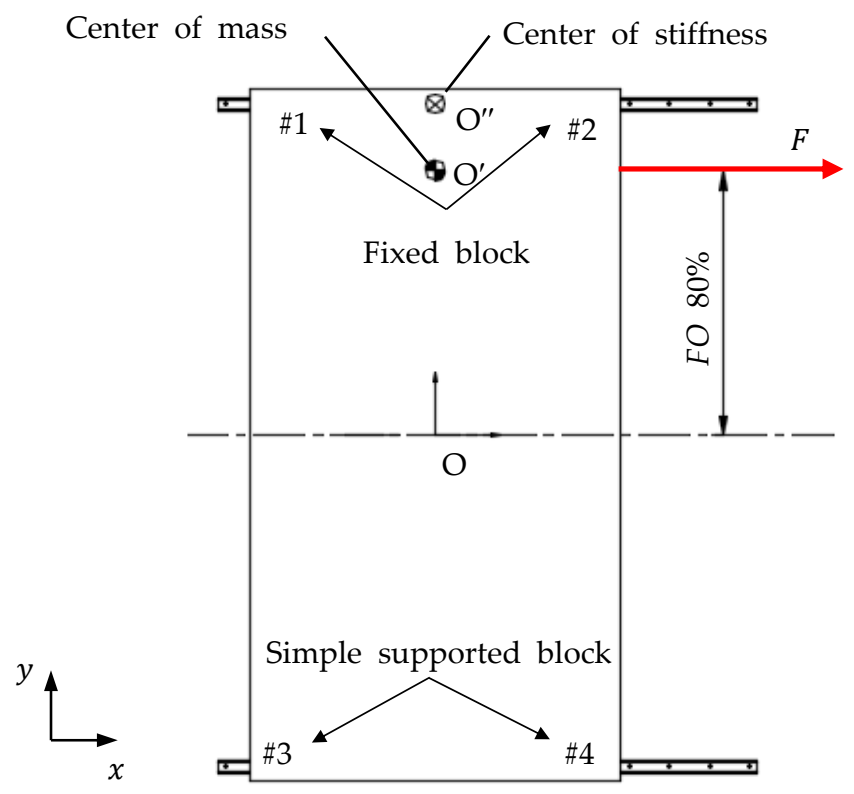

Figure 21. Modified platform for minimization of yaw angle. 


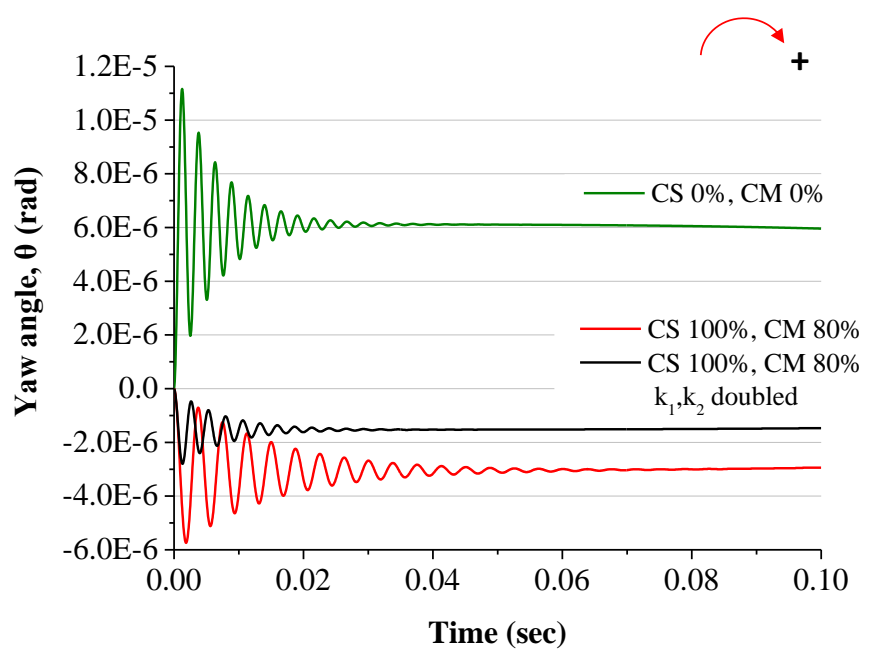

Figure 22. Modified platform for minimization of yaw angle.

Figure 22 shows three yaw angle waveforms for the deceleration stage of the trapezoidal motion profile for the No. 4 platform at $80 \%$ FO. The original model with zero values of CS and CM has clockwise yaw angle. An improved model with CS of $100 \%$ and CM of $80 \%$ has counterclockwise yaw angle half the magnitude of the original model. After the stiffness of $k_{1}$ and $k_{2}$ are doubled, the magnitude of yaw angle is further reduced to half.

\section{Design Improvement of X-Y Stage}

The results in the previous section have shown that the yaw angle of the stage decreases as the $l / w$ ratio increases, the parallelism of the LM Guides becomes better, and the platform's centers of stiffness and mass come closer to the drive line of linear actuator. The position repeatability of the stage with respect to the reciprocating motion becomes better with reduced yaw angle.

The PCB electrical test machine has an $x-y$ stage with offset driven moving platforms. In particular, it has disadvantageous spatial constraints against precision driving with the aspect ratio $l / w$ of 0.41 and $F O 80 \%$.

To overcome this challenge, the following design improvements are proposed. First, maximize $k_{1}$ and $k_{2}$ while keeping $k_{3}=k_{4}=0$ with simple support of the blocks on rail \#2. For this, THK's HSR35L and HSR20L were selected for rail \#1 and rail \#2, respectively.

Furthermore, to best match the center of mass with the driveline, a balancing weight was added to the base of moving platform assembly as shown in Figure 23. Since the platform moves along the $y$-axis with a stroke of 300mm, the CM fluctuates between 60 71\%. The linear drive actuator system was composed of a $20 \mathrm{~mm}$ lead ball screw, a 750w servo motor, and a linear encoder.

Table 9 shows the results of the yaw error calculated using Equation (10) for the improvements.

Table 9. Platform dimensions and predicted yaw error for the improved x-axis stage 


\begin{tabular}{ccccccc}
\hline \hline Axis & $\begin{array}{c}\text { Length } \\
(\mathbf{m m})\end{array}$ & $\begin{array}{c}\text { Width } \\
(\mathbf{m m})\end{array}$ & $l / w$ & $\begin{array}{c}\text { Balancing } \\
\text { weight }(\mathbf{k g})\end{array}$ & $\begin{array}{c}\text { FO } \\
(\%)\end{array}$ & $\begin{array}{c}\text { Yaw error } \\
(\mu \mathrm{m})\end{array}$ \\
\hline$x$ & 370 & 895 & 0.41 & 17.8 & 80 & \pm 0.67 \\
\hline \hline
\end{tabular}

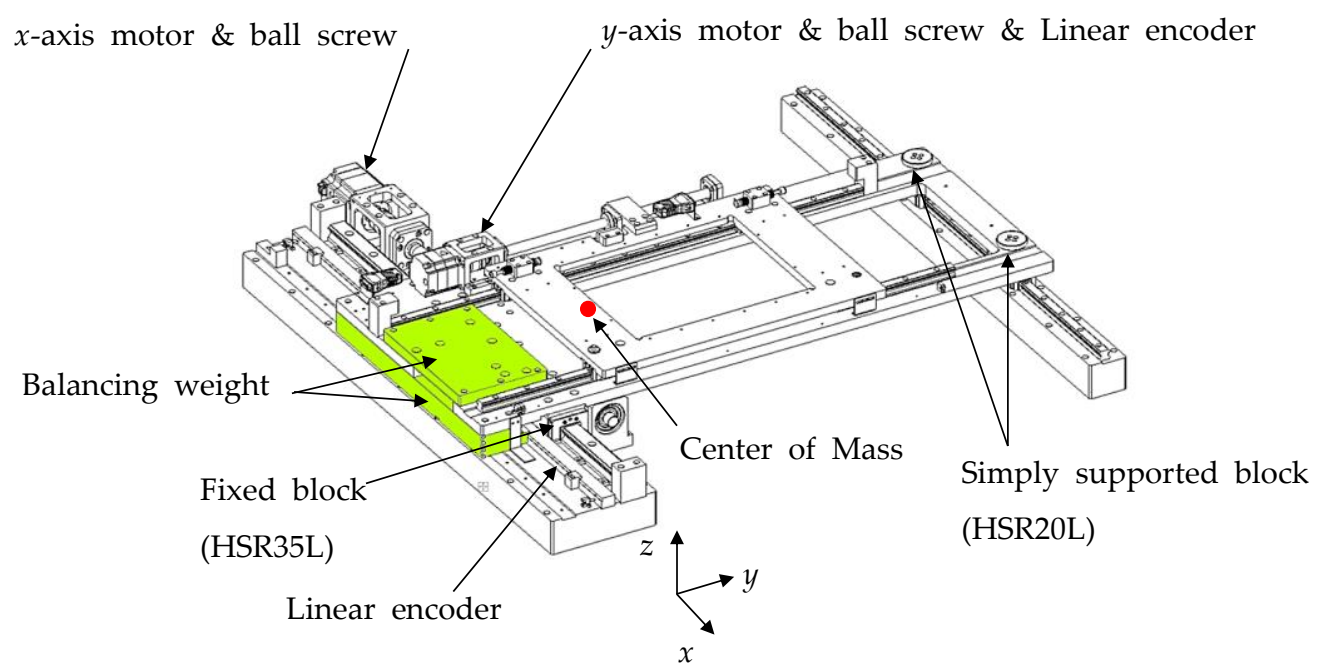

Figure 23. Design of 2-axis linear stage with balancing weight.

Since the predicted yaw error is below the target repeatability of $\pm 1.0 \mu \mathrm{m}$, the detailed design of the $\mathrm{x}$-axis was performed. The improved design of the $\mathrm{x}-\mathrm{y}$ stage is shown in Figure 22. Figure 23 shows the details of the simply supported blocks in Figure 22. In Figure 23, the platform was mounted onto the support plate, and a pin was assembled in the platform hole. A urethane ring was inserted between the pin and the platform hole to absorb the parallelism error of the LM Guide to prevent any constraint on the platform. A pin top block was utilized to constrain the $\mathrm{z}$ direction motion of the platform. The pin length was processed and assembled by approximately $10 \mu \mathrm{m}$ longer than the platform thickness.

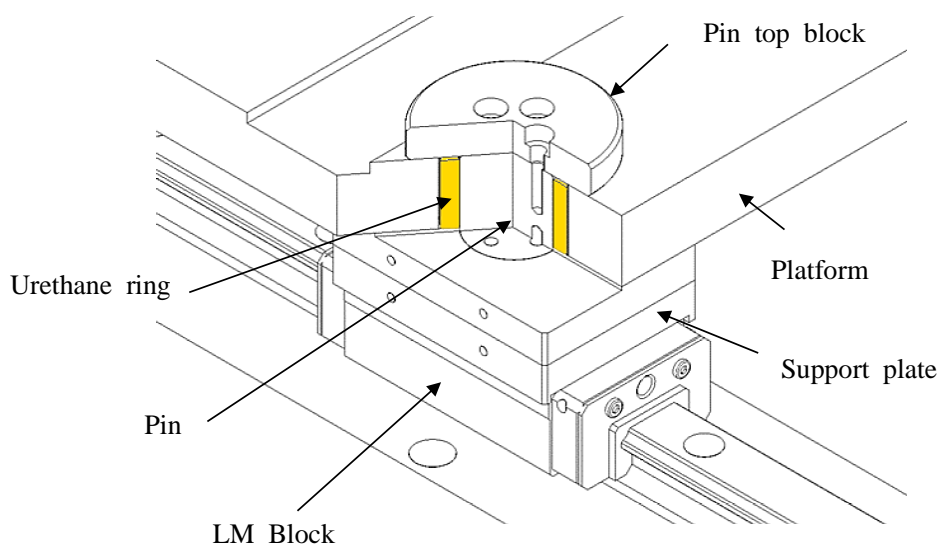

Figure 24. Sectional view of the simply supported blocks in Figure 22. 
The manufactured test bed in Figure 25 includes an improved $x-y$ linear stage and a vision camera. Artifact glass in Figure 26 was utilized for position calibration and repeatability test.

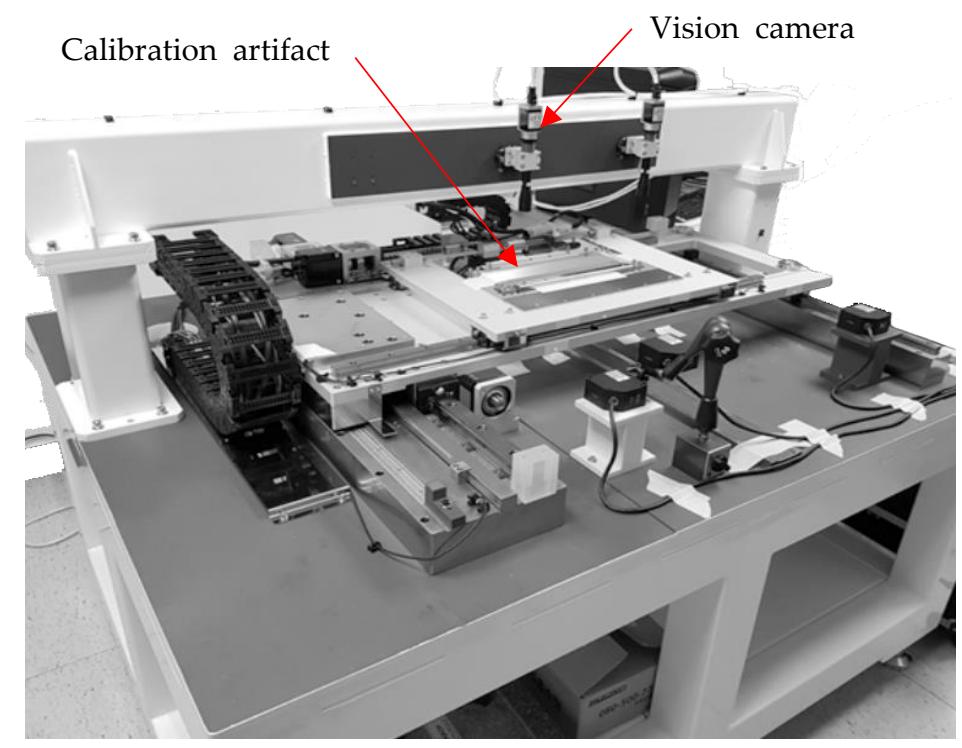

Figure 25. Test bed for improved $x-y$ linear stage

Calibration artifacts were precisely printed to be $0.5 \mathrm{~mm}$ in diameter at $5 \mathrm{~mm}$ intervals by light exposure to glass.

The position compensation of the $x-y$ stage was performed recognizing this circular dot using the vision camera.

The $x-y$ stage started from the origin to a path passing through 15 points on the artifact glass, as shown in Figure 26, according to the actual electrical test machine operations. The cycles were repeated 25 times. The $x$ and $y$ data of each point were gathered through a camera. Ideally, the coordinates of 15 points should be aligned to the center of the camera. The actual points deviated from the center due to the errors from the stage.

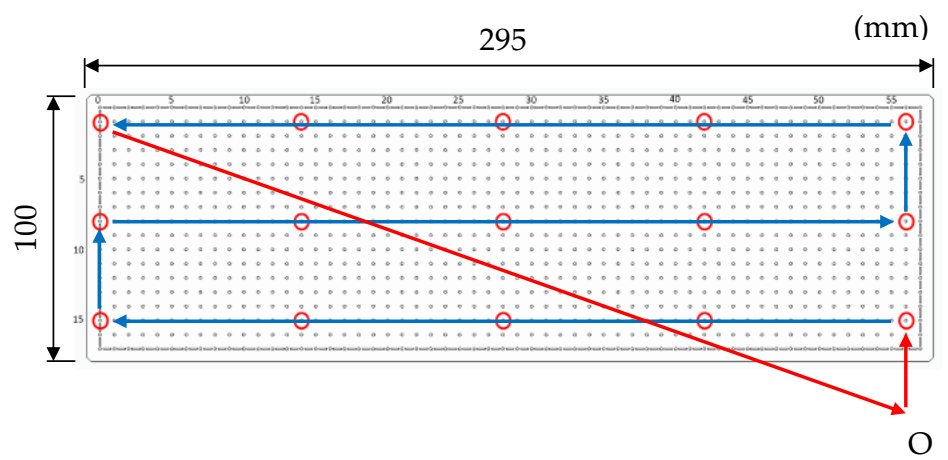

Figure 26. The calibration artifact with a pitch circle of $5 \mathrm{~mm}$ mounted on the platform of $x-y$ stage. The origin and 15 points track viewed from stationary camera.

A set of actual positions of the 15 points with respect to the center of camera for repeated 25 cycles are shown in Figure 27: (a) points without balancing weight, and (b) with the balancing weight. In Figure 27(a), the scatter in the $x$-axis was large out of $\pm 1.0 \mu \mathrm{m}$ target and in Figure 27(b), the scatter range was within the target. The six sigma analyses of the point coordinates show changes from $1.62 \sigma$ to $5.51 \sigma$ in the $x$-axis and from $3.68 \sigma$ to 
$3.21 \sigma$ in the y-axis. The process capability has been greatly improved by more than $5 \sigma$ in the $\mathrm{x}$-axis with a slight setback in the $\mathrm{y}$-axis, but still allowable beyond the practical limit of $3 \sigma$. Thus, the beneficial effects of $C S$ and $C M$ modifications were clearly verified.

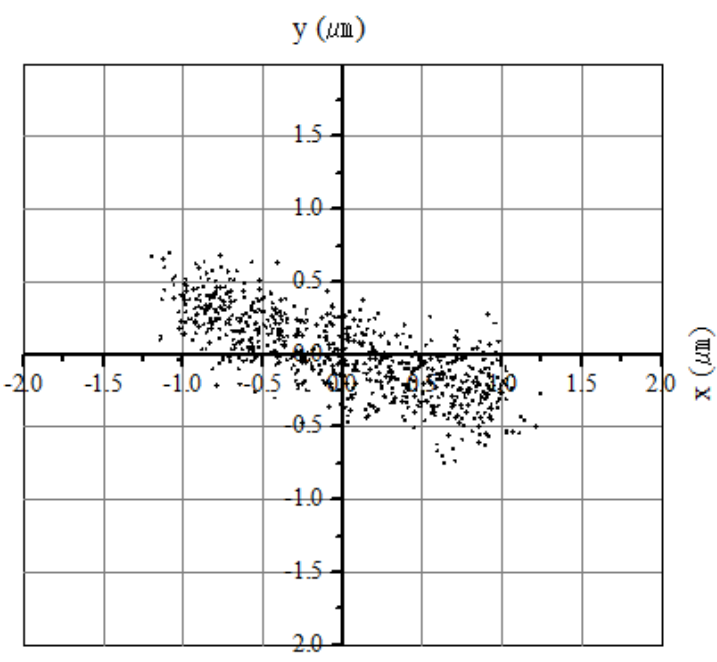

(a)

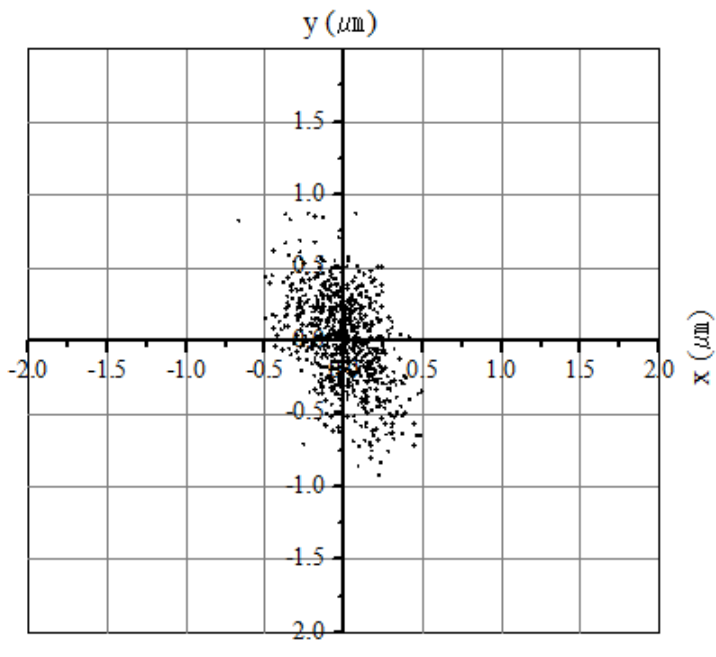

(b)

Figure 27. Markers of 15 points on calibration artifact glass for 25 cycles of repeatability test captured by camera: (a) without balancing weight and (b) with balancing weight.

The scatter patterns in Figure 27 seem to be elongated with their major axes covering the second and the fourth quadrants. This is due to one-way travel in the $x$-axis direction during the 15 points track illustrated in Figure 26. The yaw and tilt of the platform causes the elongation through the camera.

It is obvious that the application of balancing weight to the y-axis motion would produce similar results.

\section{Conclusion}

The PCB electrical test machine has an $x-y$ stage with open-frame type moving platform. It has disadvantageous conditions for precision driving with ratio $l / w$ of 0.41 and FO of $80 \%$. Practical constraints on the moving platform makes the choice unavoidable even though such a configuration is considered most unfavorable [1,2]. Practically attainable position repeatability of such configuration has been known to be in the order of tens of micrometers or more.

Since the platform yaw motion affects the stage's position repeatability significantly, its control is a crucial factor for the stage design. Yawing of moving platform is governed by various factors including the driving force offset from the platform's geometric center, the positions of stiffness center, mass center and rotation center. Other contributions are also expected from the parallelism between the two linear motion guides supporting the platform. The stiffness between the block and rail of the linear motion guides has also significant contributions.

In this work, it has been confirmed that the yawing may be minimized by reducing the distances between the linear ball screw actuator and the platform's center of mass as well as stiffness center. This may be implemented by adding proper balance weights to the platform and partially relieving constraints from one of the linear motion guides. The adverse effects of parallelism error between the two linear motion guides may be alleviated by this method. An example of design improvement along this line has been presented with experimental validation. 


\begin{abstract}
Author Contributions: Conceptualization, Cho, D. H. and Kim, K. H.; methodology, Cho, D. H. and Kwon, H. C.; validation, Cho, D. H. and Kwon, H. C.; formal analysis, Cho, D. H. and Kim, K. H.; investigation, Cho, D. H. and Kwon, H. C.; data curation, Kwon, H. C.; writing-original draft preparation, Cho, D. H.; writing - review and editing, All; visualization, Cho, D. H.; supervision, Kim, K. H.; project administration, Kim, K. H.; funding acquisition, Kim, K. H. All authors have read and agreed to the published version of the manuscript.
\end{abstract}

Funding: This research was supported by the grant "Development of next-generation electrical test machine ultra-precision driving system" from Samsung Electro-Mechanics Corporation.

Conflicts of Interest: The authors declare no conflict of interest.

\title{
References
}

1. Slocum, A.H. Precision Machine Design.; Society of Manufacturing Engineers Dearborn; Michigan, USA, 1992.

2. THK, Co., Ltd., THK Linear Motion Systems Catalog 513-1K.

3. Shimizu, S. Stiffness Analysis of Linear Motion Rolling Guide. Journal of Japanese Society for Precision Engineering, 1998, vol. 64, no. 11, 1573-1576.

4. Rahmania, M.; Bleicher, F. Experimental and analytical investigations on normal and angular stiffness of linear guides in manufacturing systems, Procedia CIRP, 2016, vol. 41, 795-800.

5. Shaukharova, A.; Liang, Y.; Feng, H.; Xu, B. Study of Stiffness of Linear Guide Pairs by Experiment and FEA, World Journal of Engineering and Technology, 2016, vol. 4, 115-128.

6. Ohta, H.; Hayashi, E. Vibration of Linear Guideway type recirculating Linear Ball Bearings, Journal of Sound and Vibration, 2000, vol. 235, no. 5, 847-861.

7. Kim, H. Y.; Jeong, J. K.; Won J. J.; Jeong, J. I. A Computational Modeling Reflecting Static and Dynamic Characteristics of LM Bearing for Machine Tools, Journal of the Korean Society for Precision Engineering, 2012, vol. 29, no. 10, 1062-1069.

8. Jeong, J.; Kang E.; Jeong, J. Equivalent stiffness Modeling of Linear Motion Guideways for Stage Systems, International Journal of Precision Engineering and Manufacturing, 2014, vol. 15, no. 9, 1987-1993.

9. Chang, J. C.; Hung, J. P. Analytical and Finite Element Modeling of the Dynamic Characteristics of a Linear Feeding Stage with Different Arrangements of Rolling Guides, Mathematical Problems in Engineering, 2014, 11.

10. Kim, K. H.; Park, C. H.; Lee, H.; Kim, S. W. Analysis of the Motion Errors in Linear Motion Guide, Journal of the Korean Society of Precision Engineering, 2002, vol. 19, no. 5, 139-148.

11. Khim, G.; Park, C. H.; Oh, J. S. A Method of Calculating Motion Error in a Linear Motion Bearing Stage, The Scientific World Journal, 2015, 10.

12. Rahmania, M.; Bleicher, F. Experimental and numerical studies of the influence of geometric deviations in the performance of machine tools linear guides, Procedia CIRP, 2016, vol. 41, 818-823.

13. Khim, G.; Park, C. H.; Shamoto, E.; Kim, S. W. Prediction and compensation of motion accuracy in a linear motion bearing table, Precision Engineering, 2011, vol. 35, 393-399.

14. Li, X.; Liu, Y.; Liu, J.; Guo, H. Theory and experimental research on static stiffness of linear rolling guide, 2012 IEEE International Conference on Computer Science and Automation Engineering (CSAE), 2012, pp. 85-87.

15. Hu, C.; Zhu, Y. Self-Calibration of Two-Dimensional Precision Metrology Systems, New Trends and Developments in Metrology, 2016, 185-210.

16. Yi, Y. S.; Kim, Y. Y.; Choi, J. S.; Yoo, J. H.; Lee, D. J.; Lee, S. W.; Lee, S. J. Dynamic analysis of a linear motion guide having rolling elements for precision positioning devices, Journal of Mechanical Science and Technology, 2008, 22, 50-60.

17. Wit, C. C. D.; Lischinsky, P. Adaptive Friction Compensation with Partially Known Dynamic Friction Model, International Journal of Adaptive Control and Signal Processing, 1997, 11, 65-80.

18. Yanga, P.; Takamuraa, T.; Takahashi, S.; Takamasua, K.; Sato b, O.; Osawa b, S.; Takatsuji, T. Development of high-precision micro-coordinate measuring machine: Multiprobe measurement system for measuring yaw and straightness motion error of XY linear stage, Precision Engineering, 2011, 35, 424-430.

19. Ekinci, T.; Mayer, J. Relationships between straightness and angular kinematic errors in machines, International Journal of Machine Tools and Manufacture, 2007, vol. 47, no. 12-13, 1997-2004.

20. Choi, J. S.; Yi, Y. S.; Kim, Y. Y.; Lee, D. J.; Lee, S. J.; Yoo, J. H. Vibration Analysis and Its Application of a Linear Motion Guide Supported by Rolling Ball Bearings, Transactions of the Korean Society of Mechanical Engineers A, 2005, vol. 29 Issue 7, no. 238, $955-$ 963.

21. Cheng, D. J.; Yang, W. S.; Park, J. H.;Park, T. J.; Kim, S.J.; Kim, G. H.; Park, C. H. Friction Experiment of Linear Motion Roller Guide THK SRG25, International Journal of Precision Engineering and Manufacturing, 2014, vol. 15, no. 3, 545-551.

22. Low, K. S.; Keck, M. T. Advanced Precision Linear Stage for Industrial Automation Application, IEEE Transactions on Instrumentation and Measurement, 2003, vol. 52, no. 3.

23. Li, H.; Le, M. D.; Gong, Z. M.; Lin, W. Motion Profile Design to Reduce Residual Vibration of High-Speed Positioning Stages, IEEE/ASME Transactions on Mechatronics, 2009, vol. 14, no. 2, 264-269.

24. GEKKO, Python package. Available online: https://gekko.readthedocs.io/en/latest/index.html\# (accessed on 19 November 2021). 